Ninth International Conference on

Geometry, Integrability and Quantization

June 8-13, 2007, Varna, Bulgaria

Ivaïlo M. Mladenov and Manuel de León, Editors

SOFTEX, Sofia 2008, pp 1-21

\title{
GEOMETRY AND TOPOLOGY OF COADJOINT ORBITS OF SEMISIMPLE LIE GROUPS
}

\author{
JULIA BERNATSKA ${ }^{\dagger \ddagger}$, PETRO HOLOD ${ }^{\dagger \ddagger}$ \\ $\dagger$ Department of Physical and Mathematical Sciences, National University of \\ 'Kyiv-Mohyla Academy' 2 Skovorody Str., 04070 Kyiv, Ukraine \\ $\ddagger$ Bogolyubov Institute for Theoretical Physics of the National Academy of Sciences \\ of Ukraine 14-b Metrolohichna Str., 03680, Kyiv, Ukraine
}

\begin{abstract}
Orbits of coadjoint representations of classical compact Lie groups have a lot of applications. They appear in representation theory, geometrical quantization, theory of magnetism, quantum optics etc. As geometric objects the orbits were the subject of much study. However, they remain hard for calculation and application. We propose simple solutions for the following problems: an explicit parameterization of the orbit by means of a generalized stereographic projection, obtaining a Kählerian structure on the orbit, introducing basis two-forms for the cohomology group of the orbit.
\end{abstract}

\section{Introduction}

Orbits of coadjoint representations of semisimple Lie groups are an extremely interesting subject. These homogeneous spaces are flag manifolds. Remarkable, that the coadjoint orbits of compact groups are Kählerian manifolds. In 1950s A. Borel, R. Bott, J. L. Koszul, F. Hirzebruch et al. investigated the coadjoint orbits as complex homogeneous manifolds. It was proven that each coadjoint orbit of a compact connected Lie group G admits a canonical G-invariant complex structure and the only (within homotopies) G-invariant Kählerian metrics. Furthermore, the coadjoint orbits can be considered as fibre bundles whose bases and fibres are coadjoint orbits themselves.

Coadjoint orbits appear in many spheres of theoretical physics, for instance in representation theory, geometrical quantization, theory of magnetism, quantum optics. They serve as definitional domains in problems connected with nonlinear integrable equations (so called equations of soliton type). Since these equations 
have a wide application, the remarkable properties of coadjoint orbits interest not only mathematicians but also physicists.

It should be pointed out that much of our material is, of course, not new, but drawn from various areas of the mathematical literature. The material was collected for solving the physical problem based on a classical Heisenberg equation with $\mathrm{SU}(n)$ as a gauge group. The equation describes a behavior of magnetics with spin $s \geqslant 1$. The paper includes an investigation of geometrical and topological properties of the coadjoint orbits. We hope it fulfills a certain need. We would like to mention that we have added a number of new results (such as an explicit expression for a stereographic projection in the case of group $\mathrm{SU}(3)$ and improving the way of its computation, the idea of obtaining the Kählerian potential on an orbit, an introduction of basis two-forms for the cohomology ring of an orbit).

The paper is organized as follows. In section 2 we recall the notion of a coadjoint orbit, propose a classification of the orbits, and describe the orbit as a fibre bundle over an orbit with an orbit as a fibre. Section 3 is devoted to a generalized stereographic projection from a Lie algebra onto its coadjoint orbit, it gives a suitable complex parameterization of the orbit. As an example, we compute an explicit expression for the stereographic projection in the case of group SU(3). In section 4 we propose a way of obtaining Kählerian structures and Kählerian potentials on the orbits. Section 5 concerns a structure of the cohomology rings of the orbits and finding of G-invariant bases for the cohomology groups.

\section{Coadjoint Orbits of Semisimple Lie Groups}

We start with recalling the notion of a coadjoint orbit. Let $\mathrm{G}$ be a compact semisimple classical Lie group, $\mathfrak{g}$ denote the corresponding Lie algebra, and $\mathfrak{g}^{*}$ denote the dual space to $\mathfrak{g}$. Let $\mathrm{T}$ be the maximal torus of $\mathrm{G}$, and $\mathfrak{h}$ be the maximal commutative subalgebra (also called a Cartan subalgebra) of $\mathfrak{g}$. Accordingly, $\mathfrak{h}^{*}$ denotes the dual space to $\mathfrak{h}$.

Definition 1. The subset $\mathcal{O}_{\mu}=\left\{\mathrm{Ad}_{g}^{*} \mu \mid \forall g \in \mathrm{G}\right\}$ of $\mathfrak{g}^{*}$ is called a coadjoint orbit of $\mathrm{G}$ through $\mu \in \mathfrak{g}^{*}$.

In the case of classical Lie groups we can use the standard representations for adjoint and coadjoint operators:

$$
\operatorname{Ad}_{g} X=g X g^{-1}, X \in \mathfrak{g}, \quad \operatorname{Ad}_{g}^{*} \mu=g^{-1} \mu g, \mu \in \mathfrak{g}^{*} .
$$

Comparing these formulas one can easily see that a coadjoint orbit coincides with an adjoint one.

Define the stability subgroup at a point $\mu \in \mathfrak{g}^{*}$ as $\mathrm{G}_{\mu}=\left\{g \in \mathrm{G} \mid \operatorname{Ad}_{g}^{*} \mu=\mu\right\}$. The coadjoint operator induces a bijective correspondence between an orbit $\mathcal{O}_{\mu}$ and a coset space $G_{\mu} \backslash G$ (in the sequel, we deal with right coset spaces). 
First of all, we classify the coadjoint orbits of an arbitrary semisimple group G. Obviously, each orbit is drawn from a unique point, which we call an initial point and denote by $\mu_{0}$. The following theorem from [1] allows to restrict the region of search of an initial point.

Theorem (R. Bott). Each orbit of the coadjoint action of $\mathrm{G}$ intersects $\mathfrak{h}^{*}$ precisely in an orbit of the Weyl group.

In other words, each orbit is assigned to a finite non-empty subset of $\mathfrak{h}^{*}$. For more detail recall the notion of the Weyl group. Let $N(\mathrm{H})$ be the normalizer of a subset $\mathrm{H} \subset \mathrm{G}$ in $\mathrm{G}$, that is $N(\mathrm{H})=\left\{g \in \mathrm{H} \mid g^{-1} \mathrm{H} g=\mathrm{H}\right\}$. Let $C(\mathrm{H})$ be the centralizer of $\mathrm{H}$, that is $C(\mathrm{H})=\left\{g \in \mathrm{G} \mid g^{-1} h g=h, \forall h \in \mathrm{H}\right\}$. Obviously, $C(\mathrm{~T})=\mathrm{T}$, where $\mathrm{T}$ is the maximal torus of $\mathrm{G}$.

Definition 2. The Weyl group of $\mathrm{G}$ is the factor-group of $N(\mathrm{~T})$ over $C(\mathrm{~T})$ :

$$
W(\mathrm{G})=N(\mathrm{~T}) / C(\mathrm{~T}) .
$$

The Weyl group $W(G)$ acts transitively on $\mathfrak{h}^{*}$. The action of $W(G)$ is performed by the coadjoint operator. It is easy to show that $\mathrm{W}(\mathrm{G})$ is isomorphic to the finite group generated by reflections $w_{\alpha}$ across the hyperplanes orthogonal to simple roots $\alpha$ :

$$
w_{\alpha}(\mu)=\mu-2 \frac{\langle\mu, \alpha\rangle}{\langle\alpha, \alpha\rangle} \alpha, \quad \mu \in \mathfrak{h}^{*},
$$

where $\langle\cdot, \cdot\rangle$ denotes a bilinear form on $\mathfrak{g}^{*}$.

Definition 3. The open domain

$$
C=\left\{\mu \in \mathfrak{h}^{*} \mid\langle\mu, \alpha\rangle>0, \forall \alpha \in \Delta^{+}\right\}
$$

is called the positive Weyl chamber. Here $\Delta^{+}$denotes the set of positive roots. We call the set $\Gamma_{\alpha}=\left\{\mu \in \mathfrak{h}^{*} \mid\langle\mu, \alpha\rangle=0\right\}$ a wall of the Weyl chamber.

If we reflect the closure $\bar{C}$ of the positive Weyl chamber by elements of the Weyl group we cover $\mathfrak{h}^{*}$ overall:

$$
\mathfrak{h}^{*}=\bigcup_{w \in \mathrm{W}(\mathrm{G})} w \cdot \bar{C} .
$$

An orbit of the Weyl group W(G) is obtained by the action of $W(G)$ on a point of $\bar{C}$. In the case of group $\mathrm{SU}(3)$, two possible types of orbits of the Weyl group are shown on the root diagram (see figure 1). Black points denote intersections of a coadjoint orbit with $\mathfrak{h}^{*}$ and form an orbit of $\mathrm{W}(\mathrm{SU}(3))$. The positive Weyl chamber is filled with grey color. It has two walls: $\Gamma_{\alpha_{1}}$ and $\Gamma_{\alpha_{2}}$; they are the hyperplanes denoted by $w_{\alpha_{1}}$ and $w_{\alpha_{2}}$. At the left, one can see a generic case, when an orbit of $\mathrm{W}(\mathrm{SU}(3))$ has 6 elements. It happens if an initial point lies in the interior of the positive Weyl chamber. At the right, there is a degenerate (non-generic) case, 


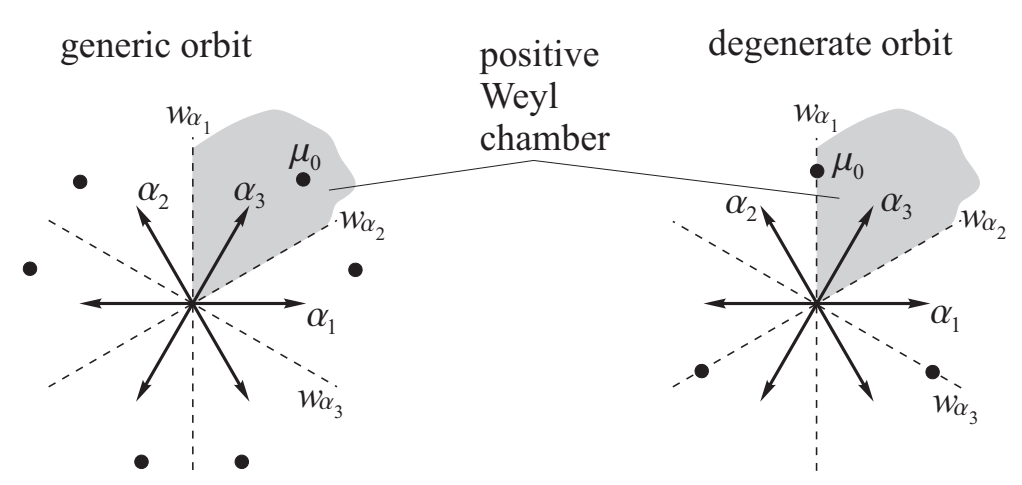

Figure 1. Root diagram for $\mathrm{SU}(3)$.

when an orbit of $\mathrm{W}(\mathrm{SU}(3))$ has 3 elements. It happens if an initial point belongs to a wall of the positive Weyl chamber.

In the both cases the closed positive Weyl chamber contains a unique point of an orbit of $\mathrm{W}(\mathrm{G})$. We obtain the following

Proposition 1. Each orbit $\mathcal{O}$ of $\mathrm{G}$ is uniquely defined by an initial point $\mu_{0} \in \mathfrak{h}^{*}$, which is located in the closed positive Weyl chamber $\bar{C}$. If $\mu_{0}$ lies in the interior of the positive Weyl chamber: $\mu_{0} \in C$, it gives rise to a generic orbit. If $\mu_{0}$ belongs to a wall of the positive Weyl chamber: $\mu_{0} \in \Gamma_{\alpha}, \alpha \in \Delta^{+}$, it gives rise to a degenerate orbit.

As mentioned above, one can define the orbit $\mathcal{O}_{\mu_{0}}$ through an initial point $\mu_{0} \in \mathfrak{h}^{*}$ by $\mathcal{O}_{\mu_{0}}=\mathrm{G}_{\mu_{0}} \backslash \mathrm{G}$. Note, that a stability subgroup $\mathrm{G}_{\mu}$ as $\mu \in \mathfrak{h}^{*}$ generically coincides with the maximal torus $\mathrm{T}$. However, if $\mu$ belongs to a degenerate orbit, then $\mathrm{G}_{\mu}$ is a lager subgroup of $\mathrm{G}$ containing $\mathrm{T}$. Therefore, we define a generic orbit by

$$
\mathcal{O}_{\mu_{0}}=\mathrm{T} \backslash \mathrm{G},
$$

and a degenerate one by

$$
\mathcal{O}_{\mu_{0}}=\mathrm{G}_{\mu_{0}} \backslash \mathrm{G}
$$

where $\mathrm{G}_{\mu_{0}} \neq \mathrm{T}, \mathrm{G}_{\mu_{0}} \supset \mathrm{T}$.

An important topological property of the coadjoint orbits is the following. Almost each orbit can be regarded as a fibre bundle over an orbit with an orbit as a fibre, except for the maximal degenerate orbits. Indeed, if there exists an initial point $\mu_{0}$ such that $\mathrm{G}_{\mu_{0}} \supset \mathrm{T}$, one can form a coset space $\mathrm{T} \backslash \mathrm{G}_{\mu_{0}}$. Thus, the orbit $\mathcal{O}_{\mu_{0}}=\mathrm{T} \backslash \mathrm{G}$ is a fibre bundle over the base $G_{\mu_{0}} \backslash G$ with the fibre $T \backslash G_{\mu_{0}}$ :

$$
\mathcal{O}_{\mu_{0}}=\mathcal{E}\left(\mathrm{G}_{\mu_{0}} \backslash \mathrm{G}, \mathrm{T} \backslash \mathrm{G}_{\mu_{0}}, \pi\right),
$$

where $\pi$ denotes a projection from the orbit onto the base. Moreover, $\mathrm{G}_{\mu_{0}} \backslash \mathrm{G}$ and $T \backslash G_{\mu 0}$ are coadjoint orbits themselves. We claim this by 
Proposition 2. Suppose $\mathcal{O}_{\mu_{0}}=\mathrm{G}_{\mu_{0}} \backslash \mathrm{G}$ is not the maximal degenerate orbit of $\mathrm{G}$. Then a subgroup $K$ such that $\mathrm{G} \supset \mathrm{K} \supset \mathrm{G}_{\mu_{0}}$ exists, and $\mathcal{O}_{\mu_{0}}$ is a fibre bundle over the base $\mathrm{K} \backslash \mathrm{G}$ with the fibre $\mathrm{G}_{\mu_{0}} \backslash \mathrm{K}$ :

$$
\mathcal{O}_{\mu_{0}}=\mathcal{E}\left(\mathrm{K} \backslash \mathrm{G}, \mathrm{G}_{\mu_{0}} \backslash \mathrm{K}, \pi\right) .
$$

We illustrate the proposition by examples.

Example 1. The group SU(2) has the only type of orbits:

$$
\mathcal{O}^{\mathrm{SU}(2)}=\frac{\mathrm{SU}(2)}{\mathrm{U}(1)} \simeq \mathbb{C P}^{1} .
$$

The group $\mathrm{SU}(3)$ has generic and degenerate orbits:

$$
\mathcal{O}^{\mathrm{SU}(3)}=\frac{\mathrm{SU}(3)}{\mathrm{U}(1) \times \mathrm{U}(1)}, \quad \mathcal{O}_{d}^{\mathrm{SU}(3)}=\frac{\mathrm{SU}(3)}{\mathrm{SU}(2) \times \mathrm{U}(1)} \simeq \mathbb{C P}^{2} .
$$

Comparing the above coset spaces we see that a generic orbit $\mathcal{O}^{\mathrm{SU}(3)}$ is a fibre bundle over a degenerate orbit $\mathcal{O}_{d}^{\mathrm{SU}(3)}$ with a fibre $\mathcal{O}^{\mathrm{SU}(2)}$ :

$$
\mathcal{O}^{\mathrm{SU}(3)}=\mathcal{E}\left(\mathcal{O}_{d}^{\mathrm{SU}(3)}, \mathcal{O}^{\mathrm{SU}(2)}, \pi\right)=\mathcal{E}\left(\mathbb{C P}^{2}, \mathbb{C P}^{1}, \pi\right) .
$$

The group SU(4) has several types of degenerate orbits. There is a list of all possible types of orbits:

$$
\begin{gathered}
\mathcal{O}^{\mathrm{SU}(4)}=\frac{\mathrm{SU}(4)}{\mathrm{U}(1) \times \mathrm{U}(1) \times \mathrm{U}(1)}, \quad \mathcal{O}_{d 1}^{\mathrm{SU}(4)}=\frac{\mathrm{SU}(4)}{\mathrm{SU}(2) \times \mathrm{U}(1) \times \mathrm{U}(1)}, \\
\mathcal{O}_{d 2}^{\mathrm{SU}(4)}=\frac{\mathrm{SU}(4)}{\mathrm{S}(\mathrm{U}(2) \times \mathrm{U}(2))}, \quad \mathcal{O}_{d 3}^{\mathrm{SU}(4)}=\frac{\mathrm{SU}(4)}{\mathrm{SU}(3) \times \mathrm{U}(1)} \simeq \mathbb{C P}^{3} .
\end{gathered}
$$

As a result, there exist several representations of a generic orbit $\mathcal{O}^{\mathrm{SU}(4)}$ as a fibre bundle. For example,

$$
\begin{aligned}
\mathcal{O}^{\mathrm{SU}(4)} & =\mathcal{E}\left(\mathcal{O}_{d 3}^{\mathrm{SU}(4)}, \mathcal{O}^{\mathrm{SU}(3)}, \pi\right)=\mathcal{E}\left(\mathbb{C P}^{3}, \mathcal{O}^{\mathrm{SU}(3)}, \pi\right) \\
\mathcal{O}^{\mathrm{SU}(4)} & =\mathcal{E}\left(\mathcal{O}_{d 2}^{\mathrm{SU}(4)}, \mathcal{O}^{\mathrm{SU}(2)}, \pi\right)=\mathcal{E}\left(\mathcal{O}_{d 2}^{\mathrm{SU}(4)}, \mathbb{C P}^{1}, \pi\right) .
\end{aligned}
$$

Example 2. In the paper we consider compact classical Lie groups. They describe linear transformations of real, complex, and quaternionic spaces. Respectively, these groups are $\mathrm{SO}(n)$ over the real field, $\mathrm{SU}(n)$ over the complex field, and $\operatorname{Sp}(n)$ over the quaternionic ring. Here we list the maximal tori of all these groups, and their representations as fibre bundles.

The maximal torus of $\mathrm{SU}(n)$ is $\mathrm{T}=\overbrace{\mathrm{U}(1) \times \mathrm{U}(1) \times \cdots \times \mathrm{U}(1)}^{n-1}$; the generic type of orbits can be represented as

$$
\mathcal{O}^{\mathrm{SU}(n)}=\mathcal{E}\left(\mathbb{C P}^{n-1}, \mathcal{O}^{\mathrm{SU}(n-1)}, \pi\right) .
$$


The maximal torus of $\mathrm{SO}(n)$ as $n=2 m$ and $n=2 m+1$ has the following form $\mathrm{T}=\underbrace{\mathrm{SO}(2) \times \mathrm{SO}(2) \times \cdots \times \mathrm{SO}(2)}_{m}$; the generic type of orbits can be represented as

$$
\begin{aligned}
\mathcal{O}^{\mathrm{SO}(2 m)} & =\mathcal{E}\left(G_{2 n ; 2}, \mathcal{O}^{\mathrm{SO}(2 m-2)}, \pi\right) \\
\mathcal{O}^{\mathrm{SO}(2 m+1)} & =\mathcal{E}\left(G_{2 n-1 ; 2}, \mathcal{O}^{\mathrm{SO}(2 m-1)}, \pi\right),
\end{aligned}
$$

where $G_{2 m ; 2}, G_{2 m-1 ; 2}$ denote real Grassman manifolds.

The maximal torus of $\operatorname{Sp}(n)$ is $\mathrm{T}=\overbrace{\mathrm{U}(1) \times \mathrm{U}(1) \times \cdots \times \mathrm{U}(1)}^{n-1}$; the generic type of orbits can be represented as

$$
\mathcal{O}^{\mathrm{Sp}(n)}=\mathcal{E}\left(\mathbb{H}^{n-1}, \mathcal{O}^{\mathrm{Sp}(n-1)}, \pi\right),
$$

where $\mathbb{H}$ denotes the quaternionic ring.

\section{Complex Parameterization of Coadjoint Orbits}

In the theory of Lie groups and Lie algebras different ways of parameterization of coadjoint orbits are available. As the most prevalent we choose a generalized stereographic projection [2]. It is named so since in the case of group $\mathrm{SU}(2)$ it gives the well-known stereographic projection onto the complex plane, which is the only orbit of $\mathrm{SU}(2)$. The generalized stereographic projection is a projection from a dual space onto a coadjoint orbit parameterized by complex coordinates.

Complex coordinates are introduced by the well-known procedure that combines Iwasawa and Gauss-Bruhat decompositions. These coordinates are often called Bruhat coordinates [3].

We start with complexifying a group $G$ in the usual way: $G^{\mathbb{C}}=\exp \{\mathfrak{g}+i \mathfrak{g}\}$. A generic orbit of $\mathrm{G}$ is defined in $\mathrm{G}^{\mathbb{C}}$ by Montgomery's diffeomorphism:

$$
\mathcal{O}=\mathrm{T} \backslash \mathrm{G} \simeq \mathrm{P} \backslash \mathrm{G}^{\mathbb{C}},
$$

where $\mathrm{P}$ denotes the minimal parabolic subgroup of $\mathrm{G}^{\mathbb{C}}$.

Equation (1) becomes apparent from the Iwasawa decomposition $G^{\mathbb{C}}=\mathrm{NAK}$, where $A \simeq \exp \{i \mathfrak{h}\}$ is the real abelian subgroup of $G^{\mathbb{C}}, N$ is a nilpotent subgroup of $G^{\mathbb{C}}$, and $K$ is the maximal compact subgroup of $G^{\mathbb{C}}$. Since we consider only compact groups $G, K$ coincides with $G$. Then the Iwasawa decomposition of $G^{\mathbb{C}}$ has the following form

$$
\mathrm{G}^{\mathbb{C}}=\mathrm{NAG} .
$$

It is easy to express $\mathrm{A}$ and $\mathrm{N}$ in terms of root vectors. Let $\Delta^{+}$be the set of positive roots $\alpha$ of $\mathrm{G}^{\mathbb{C}}$. By $X_{\alpha}, X_{-\alpha}, \alpha \in \Delta^{+}$, denote positive and negative root vectors, respectively. By $H_{\alpha}, \alpha \in \Delta^{+}$, denote the corresponding Cartan vectors, which 
form a basis for the Cartan subalgebra $\mathfrak{h}$. According to [4], we choose $X_{\alpha}$ and $X_{-\alpha}$ so that $X_{\alpha}-X_{-\alpha}, \mathrm{i}\left(X_{\alpha}+X_{-\alpha}\right) \in \mathfrak{g}$. Then

$$
\mathrm{N} \simeq \exp \left\{\sum_{\alpha \in \Delta^{+}} n_{\alpha} X_{\alpha}\right\}, n_{\alpha} \in \mathbb{C}, \quad \mathrm{A} \simeq \exp \left\{\sum_{\alpha \in \Delta^{+}} a_{\alpha} \mathrm{i} H_{\alpha}\right\}, a_{\alpha} \in \mathbb{R} .
$$

In this notation $\mathrm{P}=\mathrm{NAT}$. This makes (1) evident.

In the case of a degenerate orbit, we have the following diffeomorphism:

$$
\mathcal{O}_{\mu_{0}}=\mathrm{G}_{\mu_{0}} \backslash \mathrm{G} \simeq \mathrm{P}_{\mu_{0}} \backslash \mathrm{G}^{\mathbb{C}},
$$

where $\mathrm{G}_{\mu_{0}}$ is the stability subgroup and $\mathrm{P}_{\mu_{0}}$ is the parabolic subgroup with respect to $\mathcal{O}_{\mu_{0}}$. Then $\mathrm{P}_{\mu_{0}}=\mathrm{NAG}_{\mu_{0}}$, that proves (2).

On the other hand, G admits a Gauss decomposition (for the generic type of orbits):

$$
\mathrm{G}^{\mathbb{C}}=\mathrm{NT}^{\mathbb{C}} \mathrm{Z}
$$

where $\mathrm{T}^{\mathbb{C}}$ is the maximal torus of $\mathrm{G}^{\mathbb{C}}$, and $\mathrm{T}^{\mathbb{C}}=\mathrm{AT}$ in the above notation; $\mathrm{N}$ and $\mathrm{Z} \simeq \mathrm{N}^{*}$ are nilpotent subgroups of $\mathrm{G}^{\mathbb{C}}$ normalized by $\mathrm{T}^{\mathbb{C}}$. In terms of the root vectors introduced above

$$
\mathrm{Z}=\exp \left\{\sum_{\alpha \in \Delta^{+}} z_{\alpha} X_{-\alpha}\right\}, \quad z_{\alpha} \in \mathbb{C} .
$$

After [4] we call $a_{\alpha}, n_{\alpha}, z_{\alpha}$ the canonical coordinates connected with the root basis $\left\{H_{\alpha}, X_{\alpha}, X_{-\alpha} \mid \alpha \in \Delta^{+}\right\}$. These are coordinates in the group G.

A comparison of the Gauss and Iwasawa decompositions implies that the orbit $\mathcal{O}$ is diffeomorphic to the subgroup manifold $\mathrm{Z}$ :

$$
\mathcal{O} \simeq \frac{\mathrm{NAG}}{\mathrm{NAT}} \simeq \frac{\mathrm{NATZ}}{\mathrm{NAT}} \simeq \mathrm{Z}
$$

Diffeomorphism (3) asserts that one can parameterize the orbit $\mathcal{O}$ in terms of the complex coordinates $\left\{z_{\alpha}, \alpha \in \Delta^{+}\right\}$that are canonical coordinates in $\mathrm{Z}$.

However, a Gauss decomposition is local. Therefore, we use a Gauss-Bruhat decomposition instead:

$$
\mathrm{G}^{\mathbb{C}}=\bigcap_{w \in \mathrm{W}(\mathrm{G})} \mathrm{PZ} w .
$$

It gives a system of local charts on the orbit:

$$
\mathcal{O}=\mathrm{P} \backslash \mathrm{G}^{\mathbb{C}}=\bigcap_{w \in \mathrm{W}(\mathrm{G})} \mathrm{Z} w .
$$

In the case of a degenerate orbit $\mathcal{O}_{\mu_{0}}$, $\mathrm{T}$ is to be replaced by $\mathrm{G}_{\mu_{0}}$, and $\mathrm{P}$ by $\mathrm{P}_{\mu_{0}}$. It is sufficient to take the intersection over $w \in \mathrm{W}\left(\mathrm{G}_{\mu_{0}}\right) \backslash \mathrm{W}(\mathrm{G})$ in (4). Furthermore, in this case, $\mathrm{Z}$ has a less number of coordinates. 
Proposition 3. Each orbit $\mathcal{O}$ of a compact semisimple Lie group $\mathrm{G}$ is locally parameterized in terms of the canonical coordinates $\left\{z_{\alpha}, \alpha \in \Delta^{+}\right\}$in a nilpotent subgroup $\mathrm{Z}$ of $\mathrm{G}^{\mathbb{C}}$ according to (4).

Now we apply the above scheme to compact classical Lie groups, namely $\mathrm{SO}(n)$, $\mathrm{SU}(n), \operatorname{Sp}(n)$. The scheme consists of several steps. First we parameterize the subgroups $\mathrm{N}, \mathrm{A}$, and the group $\mathrm{G}$ in terms of $\left\{z_{\alpha}, \alpha \in \Delta^{+}\right\}$. Secondly, we choose an initial point $\mu_{0}$ in the positive closed Weyl chamber $\bar{C}$ and generate an orbit $\mathcal{O}_{\mu_{0}}$ by the dressing formula

$$
\mu=g^{-1} \mu_{0} g, \quad g \in \mathrm{G} .
$$

That gives a parameterization on one of the charts covering the orbit. Finally, we extend the parameterization to all other charts by the action of elements of the Weyl group of $\mathrm{G}$. We consider the scheme in detail.

Step 1. Being a finite group, each classical Lie group has a matrix representation. Let $\hat{a}$ be the matrix representing an element $a$. An Iwasawa decomposition of $\hat{z} \in \mathrm{Z}$ has the following form:

$$
\hat{z}=\hat{n} \hat{a} \hat{k}, \quad \hat{n} \in \mathrm{N}, \quad \hat{a} \in \mathrm{A}, \quad \hat{k} \in \mathrm{G} .
$$

One has to solve (5) in terms of the complex coordinates $z_{\alpha}$ that appear as entries of the matrix $\hat{z}$. The following transformation of (5) makes the computation easier

$$
\hat{z} \hat{z}^{*}=\hat{n} \hat{a} \hat{k} \hat{k}^{*} \hat{a}^{*} \hat{n}^{*}=\hat{n} \hat{a}^{2} \hat{n}^{*}
$$

where $\hat{k}^{*}$ denotes the hermitian conjugate of $\hat{k}$. Indeed, $\hat{k} \hat{k}^{*}=e$ for all of the mentioned groups. This is evident, if one considers the conjugation over the complex field in the case of $\mathrm{SU}(n)$, and over the quaternionic ring in the case of $\operatorname{Sp}(n)$. If $\hat{k} \in \mathrm{SO}(n)$ one has $\hat{k}^{*}=\hat{k}^{T}$, and the equality $\hat{k} \hat{k}^{*}=e$ is obvious. Moreover, it can easily be checked that $\hat{a} \hat{a}^{*}=\hat{a}^{2}$. When $\hat{n}$ and $\hat{a}$ are parameterized in terms of $\left\{z_{\alpha}\right\}$, the matrix $\hat{k}(z)$ is computed by the formula

$$
\hat{k}(z)=\hat{a}^{-1}(z) \hat{n}^{-1}(z) \hat{z} .
$$

Here we obtain complex parameterizations of $\mathrm{N}, \mathrm{A}, \mathrm{G}$ for all classical compact groups of small dimensions.

Example 3. In the case of group $\mathrm{SU}(n)$, the corresponding complexified group is $\mathrm{SL}(n, \mathbb{C})$. The subgroup $\mathrm{N}$ consists of complex upper triangular matrices with ones on the diagonal, the subgroup $\mathrm{Z}$ consists of complex low triangular matrices with ones on the diagonal, the subgroup A contains real diagonal matrices $\hat{a}=$ $\operatorname{diag}\left(r_{1}, r_{2}, \ldots, r_{n}\right)$ such that $\prod_{i=1}^{n} r_{i}=1$. 
Decomposition (5) for a generic orbit $\mathcal{O}^{\mathrm{SU}(3)}$ gets the form

$$
\left(\begin{array}{ccc}
1 & 0 & 0 \\
z_{1} & 1 & 0 \\
z_{3} & z_{2} & 1
\end{array}\right)=\left(\begin{array}{ccc}
1 & n_{1} & n_{3} \\
0 & 1 & n_{2} \\
0 & 0 & 1
\end{array}\right)\left(\begin{array}{ccc}
\frac{1}{r_{1}} & 0 & 0 \\
0 & \frac{r_{1}}{r_{2}} & 0 \\
0 & 0 & r_{2}
\end{array}\right) \hat{u}, \quad \hat{u} \in \mathrm{SU}(3),
$$

whence it follows

$$
\begin{gathered}
r_{1}^{2}=1+\left|z_{1}\right|^{2}+\left|z_{3}-z_{1} z_{2}\right|^{2}, \quad r_{2}^{2}=1+\left|z_{2}\right|^{2}+\left|z_{3}\right|^{2} \\
n_{1}=\frac{1}{r_{1}^{2}}\left(\bar{z}_{1}\left(1+\left|z_{2}\right|^{2}\right)-z_{2} \bar{z}_{3}\right), \quad n_{2}=\frac{1}{r_{2}^{2}}\left(\bar{z}_{2}+z_{1} \bar{z}_{3}\right), \quad n_{3}=\frac{\bar{z}_{3}}{r_{2}^{2}} .
\end{gathered}
$$

The dressing matrix $\hat{u}$ is

$$
\hat{u}=\left(\begin{array}{ccc}
\frac{1}{r_{1}} & -\frac{\bar{z}_{1}}{r_{1}} & -\frac{\bar{z}_{3}-\bar{z}_{1} \bar{z}_{2}}{r_{1}} \\
\frac{z_{1}\left(1+\left|z_{2}\right|^{2}\right)-z_{3} \bar{z}_{2}}{r_{1} r_{2}} & \frac{1+\left|z_{3}\right|^{2}-z_{1} z_{2} \bar{z}_{3}}{r_{1} r_{2}} & -\frac{\bar{z}_{2}+z_{1} \bar{z}_{3}}{r_{1} r_{2}} \\
\frac{z_{3}}{r_{2}} & \frac{z_{2}}{r_{2}} & \frac{1}{r_{2}}
\end{array}\right) .
$$

The case of a degenerate orbit $\mathcal{O}_{d}^{\mathrm{SU}(3)}$ is derived from the above by assigning $z_{1}=0$, or $z_{2}=0$.

Example 4. In the case of group $\operatorname{Sp}(n)$, the complexified group is $\operatorname{Sp}(n, \mathbb{C})$. The both groups describe linear transformations of the quaternionic vector space $\mathbb{H}^{n}$. Therefore, it is suitable to operate with quaternions instead of complex numbers. Each quaternion $q$ is determined by two complex numbers $z_{1}, z_{2}$ as $q=z_{1}+z_{2} \mathbf{j}$. The quaternionic conjugate of $q$ is $\bar{q}=\bar{z}_{1}-\mathbf{j} \bar{z}_{2}$, where $\bar{z}_{1}, \bar{z}_{2}$ are the complex conjugates of $z_{1}, z_{2}$. Several useful relations are available:

$$
\mathbf{j} z=\bar{z} \mathbf{j}, \quad \overline{z+w}=\bar{z}+\bar{w}, \quad \overline{z \cdot w}=\bar{w} \cdot \bar{z},
$$

where $z, w \in \mathbb{C}$.

The subgroups $\mathrm{N}, \mathrm{Z}$ have the same representatives as in the case of group $\mathrm{SU}(n)$, but over the quaternionic ring. The subgroup A consists of real diagonal matrices with the same property as in the case of $\mathrm{SU}(n)$.

We start with the simplest group $\operatorname{Sp}(2)$. Suppose $v, q \in \mathbb{H}$ such that $v=n_{1}+n_{2} \mathbf{j}$, $q=z_{1}+z_{2} \mathbf{j}$, where $n_{1}, n_{2}, z_{1}, z_{2} \in \mathbb{C}$. Decomposition (5) for an orbit $\mathcal{O}^{\mathrm{Sp}(2)}$ gets the following form

$$
\left(\begin{array}{ll}
1 & 0 \\
q & 1
\end{array}\right)=\left(\begin{array}{ll}
1 & v \\
0 & 1
\end{array}\right)\left(\begin{array}{ll}
\frac{1}{r} & 0 \\
0 & r
\end{array}\right) \hat{p}, \quad \hat{p} \in \operatorname{Sp}(2),
$$

whence it follows $r^{2}=1+|q|^{2}, v=\bar{q} / r^{2}$, or in terms of complex coordinates:

$$
r^{2}=\left|z_{1}\right|^{2}+\left|z_{2}\right|^{2}, \quad n_{1}=\frac{\bar{z}_{1}}{r^{2}}, \quad n_{2}=-\frac{z_{2}}{r^{2}} .
$$


The dressing matrix $\hat{p}$ is

$$
\hat{p}=\frac{1}{\sqrt{\left|z_{1}\right|^{2}+\left|z_{2}\right|^{2}}}\left(\begin{array}{cc}
1 & -\bar{z}_{1}+\mathbf{j} \bar{z}_{2} \\
z_{1}+z_{2} \mathbf{j} & 1
\end{array}\right) .
$$

In the case of group $\mathrm{Sp}(3)$, we perform all computations in terms of quaternions. Suppose $q_{1}=z_{1}+z_{2} \mathbf{j}, q_{2}=z_{3}+z_{4} \mathbf{j}, q_{3}=z_{5}+z_{6} \mathbf{j}, v_{1}=n_{1}+n_{2} \mathbf{j}, v_{2}=n_{3}+n_{4} \mathbf{j}$, $v_{3}=n_{5}+n_{6} \mathbf{j}$. Then, for a generic orbit $\mathcal{O}^{\mathrm{Sp}(3)}$, one obtains

$$
\left(\begin{array}{ccc}
1 & 0 & 0 \\
q_{1} & 1 & 0 \\
q_{3} & q_{2} & 1
\end{array}\right)=\left(\begin{array}{ccc}
1 & v_{1} & v_{3} \\
0 & 1 & v_{2} \\
0 & 0 & 1
\end{array}\right)\left(\begin{array}{ccc}
\frac{1}{r_{1}} & 0 & 0 \\
0 & \frac{r_{1}}{r_{2}} & 0 \\
0 & 0 & r_{2}
\end{array}\right) \hat{p}, \quad \hat{p} \in \operatorname{Sp}(3)
$$

whence it follows

$$
\begin{gathered}
r_{1}^{2}=1+\left|q_{1}\right|^{2}+\left|q_{3}-q_{2} q_{1}\right|^{2}, \quad r_{2}^{2}=1+\left|q_{2}\right|^{2}+\left|q_{3}\right|^{2} \\
v_{1}=\frac{1}{r_{1}^{2}}\left(\bar{q}_{1}\left(1+\left|q_{2}\right|^{2}\right)-\bar{q}_{3} q_{2}\right), \quad v_{2}=\frac{1}{r_{2}^{2}}\left(\bar{q}_{2}+q_{1} \bar{q}_{3}\right), \quad v_{3}=\frac{\bar{q}_{3}}{r_{2}^{2}} .
\end{gathered}
$$

The dressing matrix $\hat{p}$ is

$$
\hat{p}=\left(\begin{array}{ccc}
\frac{1}{r_{1}} & -\frac{\bar{q}_{1}}{r_{1}} & -\frac{\bar{q}_{3}-\bar{q}_{1} \bar{q}_{2}}{r_{1}} \\
\frac{q_{1}\left(1+\left.||_{2}\right|^{2}\right)-\bar{q}_{2} q_{3}}{r_{1} r_{2}} & \frac{1+\left|q_{3}\right|^{2}-q_{1} \bar{q}_{3} q_{2}}{r_{1} r_{2}} & -\frac{\bar{q}_{2}+q_{1} \bar{q}_{3}}{r_{1} r_{2}} \\
\frac{q_{3}}{r_{2}} & \frac{q_{2}}{r_{2}} & \frac{1}{r_{2}}
\end{array}\right)
$$

The case of $\operatorname{Sp}(n)$ in terms of quaternions is very similar to the case of $\mathrm{SU}(n)$. The only warning is that the multiplication of quaternions is not commutative.

Example 5. In the case of group $\mathrm{SO}(n)$, the corresponding complexified group is $\mathrm{SO}(n, \mathbb{C})$. Representatives of the subgroups $\mathrm{N}$ and $\mathrm{Z}$ have not so clear structure as for groups $\mathrm{SU}(n)$ and $\mathrm{Sp}(n)$. The real abelian subgroup A consists of blockdiagonal matrices $\hat{a}=\operatorname{diag}\left(A_{1}, A_{2}, \ldots, A_{m}\right)$ in the case of group $\mathrm{SO}(2 m)$, and $\hat{a}=\operatorname{diag}\left(A_{1}, A_{2}, \ldots, A_{m}, 1\right)$ in the case of group $\mathrm{SO}(2 m+1)$. Here

$$
A_{i}=\left(\begin{array}{cc}
\cosh a_{i} & -\mathrm{i} \sinh a_{i} \\
\mathrm{i} \sinh a_{i} & \cosh a_{i}
\end{array}\right) .
$$

Consider the group $\mathrm{SO}(3)$. The only type of orbits is $\mathcal{O}^{\mathrm{SO}(3)}=\mathrm{SO}(2) \backslash \mathrm{SO}(3)$. In this case decomposition (5) gets the form

$$
\left(\begin{array}{ccc}
1-\frac{z^{2}}{2} & -\frac{\mathrm{i} z^{2}}{2} & -z \\
-\frac{\mathrm{i} z^{2}}{2} & 1+\frac{z^{2}}{2} & -\mathrm{i} z \\
z & \mathrm{i} z & 1
\end{array}\right)=\left(\begin{array}{ccc}
1-\frac{n^{2}}{2} & \frac{\mathrm{i} n^{2}}{2} & n \\
\frac{\mathrm{i} n^{2}}{2} & 1+\frac{n^{2}}{2} & -\mathrm{i} n \\
-n & \mathrm{i} n & 1
\end{array}\right)\left(\begin{array}{ccc}
\cosh a & -\mathrm{i} \sinh a & 0 \\
\mathrm{i} \sinh a & \cosh a & 0 \\
0 & 0 & 1
\end{array}\right) \hat{o}
$$


where $\hat{o} \in \mathrm{SO}(3)$, and $a, n, z$ are canonical coordinates in the group. One easily computes the following

$$
e^{a}=1+|z|^{2}, \quad n=\frac{\bar{z}}{1+|z|^{2}}
$$

The dressing matrix $\hat{o}$ is

$$
\hat{o}=\left(\begin{array}{ccc}
\frac{2-z^{2}-\bar{z}^{2}}{2\left(1+|z|^{2}\right)} & \frac{\mathrm{i}\left(\bar{z}^{2}-z^{2}\right)}{2\left(1+|z|^{2}\right)} & -\frac{z+\bar{z}}{1+||^{2}} \\
\frac{\mathrm{i}\left(\bar{z}^{2}-z^{2}\right)}{2\left(1+|z|^{2}\right)} & \frac{2+z^{2}+\bar{z}^{2}}{2\left(1+|z|^{2}\right)} & -\frac{\mathrm{i}(z-z \bar{z})}{1+|z|^{2}} \\
\frac{z+z \bar{z}}{1+|z|^{2}} & \frac{\mathrm{i}(z-\bar{z})}{1+|z|^{2}} & \frac{1-|z|^{2}}{1+|z|^{2}}
\end{array}\right) .
$$

We return to the scheme.

Step 2. Suppose we have some parameterization of the dual space $\mathfrak{g}^{*}$ to the algebra $\mathfrak{g}$ of a group $\mathrm{G}$. We call these parameters group coordinates. In order to parameterize an orbit of $\mathrm{G}$ we find expressions for the group coordinates in terms of the complex coordinates $\left\{z_{\alpha}, \alpha \in \Delta^{+}\right\}$. Continue the example of group $\mathrm{SU}(3)$.

Let $\lambda_{a}, a=1 . .8$, be Gell-Mann matrices, then $Y_{a}=-\frac{\mathrm{i}}{2} \lambda_{a}, a=1 . .8$, form a basis for $\mathfrak{g}^{*}$. Define a bilinear form on $\mathfrak{g}^{*}$ as $\langle A, B\rangle=-2 \operatorname{Tr} A B$. Each basis element $Y_{a}$ is assigned to a group coordinate: $\mu_{a}=\left\langle\hat{\mu}, Y_{a}\right\rangle$, where

$$
\hat{\mu}=-\frac{\mathrm{i}}{2}\left(\begin{array}{ccc}
\mu_{3}+\frac{1}{\sqrt{3}} \mu_{8} & \mu_{1}-\mathrm{i} \mu_{2} & \mu_{4}-\mathrm{i} \mu_{5} \\
\mu_{1}+\mathrm{i} \mu_{2} & -\mu_{3}+\frac{1}{\sqrt{3}} \mu_{8} & \mu_{6}-\mathrm{i} \mu_{7} \\
\mu_{4}+\mathrm{i} \mu_{5} & \mu_{6}+\mathrm{i} \mu_{7} & -\frac{2}{\sqrt{3}} \mu_{8}
\end{array}\right) .
$$

A coadjoint orbit is generated by the dressing formula:

$$
\hat{\mu}=\hat{u}^{*} \hat{\mu}_{0} \hat{u}, \quad \hat{\mu}_{0} \in \mathfrak{h}^{*},
$$

where $\hat{\mu}_{0}$ is an initial point. As shown in section 2, each orbit is uniquely defined by a point of the closed positive Weyl chamber. Let simple roots of $\mathfrak{s u}(3)$ be as follows: $\hat{\alpha}_{1}=\operatorname{diag}(\mathrm{i},-\mathrm{i}, 0)$ and $\hat{\alpha}_{2}=\operatorname{diag}(0, \mathrm{i},-\mathrm{i})$. The closed positive Weyl chamber is the set of points $\hat{\mu}_{0}$ such that

$$
\hat{\mu}_{0}=-\frac{\mathrm{i}}{3} \xi\left(\begin{array}{ccc}
2 & 0 & 0 \\
0 & -1 & 0 \\
0 & 0 & -1
\end{array}\right)-\frac{\mathrm{i}}{3} \eta\left(\begin{array}{ccc}
1 & 0 & 0 \\
0 & 1 & 0 \\
0 & 0 & -2
\end{array}\right), \quad \xi, \eta>0 .
$$

Obviously, walls of the Weyl chamber are obtained by assigning $\xi=0$ or $\eta=0$. In this notation $\Gamma_{\alpha_{1}}=\left\{-\frac{i}{3} \eta \operatorname{diag}(1,1,-2) \mid \eta>0\right\}, \Gamma_{\alpha_{2}}=\left\{-\frac{i}{3} \xi \operatorname{diag}(2,-1,-1) \mid\right.$ $\xi>0\}$. The chosen representation of an initial point $\hat{\mu}_{0}$ is the most suitable for the further computation.

According to Proposition 1 we get a generic orbit if $\eta \neq 0$ and $\xi \neq 0$. If $\xi$ or $\eta$ vanishes, we get a degenerate one. A generic orbit is parameterized by three complex coordinates $z_{1}, z_{2}, z_{3}$. If $\xi$ vanishes, one has to assign $z_{1}=0$. If $\eta$ vanishes, 
then $z_{2}=0$. We consider the degenerate orbit through the following point

$$
\hat{\mu}_{0}=-\frac{\mathrm{i}}{3} \eta\left(\begin{array}{ccc}
1 & 0 & 0 \\
0 & 1 & 0 \\
0 & 0 & -2
\end{array}\right) .
$$

One can attach some physical meaning to nonzero entries of the initial point $\hat{\mu}_{0}$ because of its diagonal form. For in quantum mechanics diagonal matrices represent observable variables. Suppose $\hat{\mu}_{0}$ is the value of $\hat{\mu}$ at the infinity: $\hat{\mu}_{0}=\hat{\mu}(\infty)$. The diagonal entries are expressed in terms of the group coordinates $\mu_{3}$ and $\mu_{8}$; we fix their values at the infinity: $\mu_{3}(\infty)=m, \mu_{8}(\infty)=q$. Then

$$
\eta=-\frac{1}{2}(m-\sqrt{3} q), \quad \xi=m .
$$

Suppose the group $\mathrm{SU}(3)$ describes a magnetic with spin 1 . Then $m$ serves as a projection of magnetic moment (magnetization) of the magnetic, and $q$ serves as a projection of quadrupole moment.

The dressing procedure gives the following explicit expression for the generalized stereographic projection onto a generic orbit of $\mathrm{SU}(3)$ :

$$
\begin{aligned}
& \mu_{1}=-\frac{\eta}{r_{2}^{2}}\left(\bar{z}_{2} z_{3}+z_{2} \bar{z}_{3}\right)-\frac{\xi}{r_{1}^{2}}\left(z_{1}+\bar{z}_{1}\right) \\
& \mu_{2}=\frac{\mathrm{i} \eta}{r_{2}^{2}}\left(\bar{z}_{2} z_{3}-z_{2} \bar{z}_{3}\right)+\frac{\mathrm{i} \xi}{r_{1}^{2}}\left(z_{1}-\bar{z}_{1}\right) \\
& \mu_{3}=\frac{\eta}{r_{2}^{2}}\left(\left|z_{2}\right|^{2}-\left|z_{3}\right|^{2}\right)+\frac{\xi}{r_{1}^{2}}\left(1-\left|z_{1}\right|^{2}\right) \\
& \mu_{4}=-\frac{\eta}{r_{2}^{2}}\left(z_{3}+\bar{z}_{3}\right)-\frac{\xi}{r_{1}^{2}}\left(z_{3}-z_{1} z_{2}+\bar{z}_{3}-\bar{z}_{1} \bar{z}_{2}\right) \\
& \mu_{5}=\frac{\mathrm{i} \eta}{r_{2}^{2}}\left(z_{3}-\bar{z}_{3}\right)+\frac{\mathrm{i} \xi}{r_{1}^{2}}\left(z_{3}-z_{1} z_{2}-\left(\bar{z}_{3}-\bar{z}_{1} \bar{z}_{2}\right)\right) \\
& \mu_{6}=-\frac{\eta}{r_{2}^{2}}\left(z_{2}+\bar{z}_{2}\right)+\frac{\xi}{r_{1}^{2}}\left(\bar{z}_{1}\left(z_{3}-z_{1} z_{2}\right)+z_{1}\left(\bar{z}_{3}-\bar{z}_{1} \bar{z}_{2}\right)\right) \\
& \mu_{7}=\frac{\mathrm{i} \eta}{r_{2}^{2}}\left(z_{2}-\bar{z}_{2}\right)-\frac{\mathrm{i} \xi}{r_{1}^{2}}\left(\bar{z}_{1}\left(z_{3}-z_{1} z_{2}\right)-z_{1}\left(\bar{z}_{3}-\bar{z}_{1} \bar{z}_{2}\right)\right) \\
& \sqrt{3} \mu_{8}=\frac{\eta}{r_{2}^{2}}\left(2-\left|z_{2}\right|^{2}-\left|z_{3}\right|^{2}\right)+\frac{\xi}{r_{1}^{2}}\left(1+\left|z_{1}\right|^{2}-2\left|z_{3}-z_{1} z_{2}\right|^{2}\right),
\end{aligned}
$$

where

$$
r_{1}^{2}=1+\left|z_{1}\right|^{2}+\left|z_{3}-z_{1} z_{2}\right|^{2}, \quad r_{2}^{2}=1+\left|z_{2}\right|^{2}+\left|z_{3}\right|^{2} .
$$

Obviously, all expressions can be divided into two parts: with the coefficients $\eta$ and $\xi$. These parts correspond to the basis matrices in (6). 
For the stereographic projection onto a degenerate orbit through $\hat{\mu}_{0}$ chosen above one has to assign $\xi=0, z_{1}=0$ in (7).

Step 3. Parameterization (7) is available on the coordinate chart containing the point $\left(z_{1}=0, z_{2}=0, z_{3}=0\right)$. By the action of elements of the Weyl group one obtains parameterizations on all other charts. The Weyl group is generated by reflections across the hyperplanes orthogonal to simple roots. In the case of group $\mathrm{SU}(3)$, these reflections are represented by the following matrices

$$
\hat{w}_{1}=\left(\begin{array}{ccc}
0 & 1 & 0 \\
1 & 0 & 0 \\
0 & 0 & -1
\end{array}\right), \quad \hat{w}_{2}=\left(\begin{array}{ccc}
-1 & 0 & 0 \\
0 & 0 & 1 \\
0 & 1 & 0
\end{array}\right) .
$$

The action of $\hat{w}_{1}$ transforms the chart with coordinates (7) onto another one by the following change of coordinates:

$$
\left(z_{1}, z_{2}, z_{3}\right) \mapsto\left(z_{1}^{\prime}, z_{2}^{\prime}, z_{3}^{\prime}\right), \quad z_{1}^{\prime}=\frac{1}{z_{1}}, z_{2}^{\prime}=-z_{3}, z_{3}^{\prime}=-z_{2} .
$$

This chart contains the point $\left(z_{1}=\infty, z_{2}=0, z_{3}=0\right)$. The action of $\hat{w}_{2}$ transforms coordinates ( 7 ) by the following change of coordinates:

$$
\left(z_{1}, z_{2}, z_{3}\right) \mapsto\left(z_{1}^{\prime}, z_{2}^{\prime}, z_{3}^{\prime}\right), \quad z_{1}^{\prime}=-\left(z_{3}-z_{1} z_{2}\right), z_{2}^{\prime}=\frac{1}{z_{2}}, z_{3}^{\prime}=-\frac{z_{3}}{z_{2}} .
$$

The latter chart contains the point $\left(z_{1}=0, z_{2}=\infty, z_{3}=0\right)$.

Evidently, the other elements of $\mathrm{W}(\mathrm{SU}(3))$ are $\hat{e}, \hat{w}_{1} \hat{w}_{2}, \hat{w}_{2} \hat{w}_{1}, \hat{w}_{1} \hat{w}_{2} \hat{w}_{1}$. The corresponding changes of coordinates are obtained by sequential actions of the two described above.

\section{Kählerian Structure on Coadjoint Orbits}

The perfect property of coadjoint orbits of compact semisimple Lie groups is the following. Each orbit is simultaneously a Riemannian manifold and a symplectic one. A Riemanian metrics and the matched symplectic form together are called a Kählerian structure. A. Borel [5] proved the following

Proposition 4. Suppose G is a semisimple compact Lie group. Then each orbit of $\mathrm{G}$ admits a complex analytic Kählerian structure invariant under the group $\mathrm{G}$.

It means that each orbit possesses a hermitian Riemannian metrics, the Kählerian metrics $d s^{2}$, and the corresponding closed two-form, the Kählerian form $\omega$ :

$$
d s^{2}=\sum_{\alpha, \beta} g_{\alpha \bar{\beta}} d z_{\alpha} d \bar{z}_{\beta}, \quad \omega=\sum_{\alpha, \beta} \mathrm{i} g_{\alpha \bar{\beta}} d z_{\alpha} \wedge d \bar{z}_{\beta} .
$$


The G-invariance of a Kählerian structure means invariance under the action of G. Here we consider the action of a group as right multiplication. A Kählerian structure is determined by a Kählerian potential $\Phi$ according to the formula

$$
g_{\alpha \bar{\beta}}=\frac{\partial^{2} \Phi}{\partial z_{\alpha} \partial \bar{z}_{\beta}}, \quad \omega_{\alpha \bar{\beta}}=\mathrm{i} g_{\alpha \bar{\beta}} .
$$

The objective of this section is to obtain an expression for a Kählerian structure on a coadjoint orbit. Evidently, for this purpose it is sufficient to find a Kählerian potential, which simultaneously gives the Kählerian metrics and the Kählerian form.

On the other hand, one has the following

Proposition 5 (see [6]). If $\mathrm{G}$ is a compact semisimple Lie group, the KirillovKostant-Souriau two-form coincides with a G-invariant Kählerian form.

While we deal with compact semisimple classical Lie groups, we can use a KirillovKostant-Souriau differential form as a Kählerian form.

Define a bilinear form on $\mathfrak{g}$ as follows:

$$
\langle X, Y\rangle=\operatorname{Tr} X Y, \quad X, Y \in \mathfrak{g} .
$$

In the case of classical Lie groups, the bilinear form is proportional to the standard Killing form on $\mathfrak{g}$.

Define a vector field $\tilde{X}$ on a coadjoint orbit $\mathcal{O}$ by

$$
\tilde{X} f(\mu)=\left.\frac{d}{d \tau} f\left(\operatorname{Ad}_{\exp (\tau X)}^{*} \mu\right)\right|_{\tau=0}, \quad f \in C^{\infty}(\mathcal{O}) .
$$

One can introduce an Ad-invariant closed two-form on $\mathcal{O}$ by the formula

$$
\omega(\tilde{X}, \tilde{Y})=\langle\mu,[X, Y]\rangle, \quad X, Y \in \mathfrak{g}, \quad \mu \in \mathfrak{g}^{*} .
$$

This two-form is called a Kirillov-Kostant-Souriau form.

The straightforward way of obtaining a Kählerian form is to solve equations (8). Unfortunately, it becomes extremely complicate in dimensions greater than 3 . This way is developed by R. F. Picken in [3]. He computes Kählerian forms on flag manifolds via G-invariant one-forms in terms of Bruhat coordinates.

We return to the idea of finding a Kählerian potential instead of a Kählerian form. In general, each G-covariant real function on an orbit serves as a Kählerian potential. It turns out, that each orbit has a unique G-covariant real function, which we call a Kählerian potential on the orbit.

The same idea is used by D. V. Alekseevsky and A. M. Perelomov in [7]. In order to find potentials for all closed two-forms on orbits of group $\mathrm{GL}(n)$, they consider the real positive functions built by means of principal minors of $\hat{z} \hat{z}^{*} \in \operatorname{GL}(n)$, and 
select the functions that are G-covariant. Here we develop the idea of D. V. Alekseevsky and A. M. Perelomov, because this way allows to avoid complicate computations.

Below we prove that a Kählerian potential is determined by a one-dimensional irreducible representation of the real abelian subgroup $A$ of $G^{\mathbb{C}}$. We use the grouptheoretical approach in our proof.

Each orbit $\mathcal{O}=\mathrm{P} \backslash \mathrm{G}^{\mathbb{C}}$ is a holomorphic manifold, which admits the construction of a line bundle. Let $\left\{U_{k}\right\}$ be its atlas. An arbitrary $g_{\mathbb{C}} \in \mathrm{G}^{\mathbb{C}}$ has a decomposition

$$
g_{\mathbb{C}}=h_{k}(x) s_{k}(x), \quad x \in U_{k},
$$

where $s_{k}: U_{k} \rightarrow \mathrm{G}^{\mathbb{C}}$ is a local section of $\mathcal{O}$. If $U_{k} \cap U_{j} \neq \emptyset$, then there exists a map $s_{k j}=s_{k} \circ s_{j}^{-1}$, which is $s_{k j}: U_{k} \cap U_{j} \rightarrow \mathrm{P}$. A one-dimensional representation of the parabolic subgroup $\mathrm{P}$ of $\mathrm{G}^{\mathbb{C}}$ gives a $\mathrm{G}$-covariant function on an orbit.

Recall, that $\mathrm{P}=\mathrm{NAT}$ in the case of a generic orbit. In the case of a degenerate orbit, one has $\mathrm{P}=\mathrm{NAG}_{\mu_{0}}$, where $\mathrm{G}_{\mu_{0}}$ is the stability subgroup at an initial point $\mu_{0} \in \mathfrak{h}^{*}$ giving rise to the orbit. A one-dimensional irreducible representation is trivial on any nilpotent group. This means that the representation of $\mathrm{P}$ coincides with the representation of the maximal torus $\mathrm{T}^{\mathbb{C}}=\mathrm{AT}$ of $\mathrm{G}^{\mathbb{C}}$. Moreover, we are interested in real representations because a Kählerian potential is a real function. Consequently, the required representation is determined only by $\mathrm{A}$.

Now we build a one-dimensional irreducible representation of $T^{\mathbb{C}}$. Obviously, $\mathrm{T}^{\mathbb{C}}$ is isomorphic to a direct product of $l$ samples of the multiplicative group $\mathbb{C}^{*}=\mathbb{C} \backslash\{0\}$, where $l=\operatorname{dim} \mathrm{T}$. Let the following set of complex numbers $\left(d_{1}\right.$, $\left.d_{2}, \ldots, d_{l}\right)$ be an image of $\hat{d} \in \mathrm{T}^{\mathbb{C}}$ under the isomorphism. It is clear that the set of real numbers $\left(r_{1}, r_{2}, \ldots, r_{l}\right)$, where $r_{i}=\left|d_{i}\right|, i=1 . . l$, is an image of $\hat{a} \in \mathrm{A}$ under the isomorphism. In terms of complex coordinates $z=\left\{z_{\alpha} \mid \alpha \in \Delta^{+}\right\}$, which are canonical coordinates in $\mathrm{Z}$, an Iwasawa decomposition of any $\hat{z} \in \mathrm{Z}$ gets the form

$$
\hat{z}=\hat{n}(z) \hat{a}(z) \hat{k}(z) .
$$

Here $\hat{k}(z)$ represents a point of an orbit in terms of the complex coordinates $\left\{z_{\alpha}\right\}$; $\hat{n}(z)$ and $\hat{a}(z)$ denote matrices $\hat{n}$ and $\hat{a}$ in terms of $\left\{z_{\alpha}\right\}$. After the action of an element $g \in \mathrm{G}$ on $z$ we perform a Gauss-Bruhat decomposition:

$$
\hat{z} \hat{g}=\hat{n}_{B}(z g) \hat{d}(z g) \hat{z}_{g}, \quad \hat{n}_{B}(z g) \in \mathrm{N} .
$$

From the Iwasawa decomposition of $\hat{z}_{g}$ we have

$$
\hat{a}\left(z_{g}\right)=\hat{n}^{-1}\left(z_{g}\right) \hat{z}_{g} \hat{k}^{-1}\left(z_{g}\right) .
$$

Using (10) and (11) we get

$$
\hat{a}\left(z_{g}\right)=\hat{n}^{-1}\left(z_{g}\right) \hat{d}^{-1}(z g) \hat{n}_{B}^{-1}(z g) \hat{n}(z) \hat{a}(z) \hat{k}(z) \hat{g} \hat{k}^{-1}\left(z_{g}\right) .
$$


In order to gather nilpotent elements together we recall that the maximal torus $\mathrm{T}^{\mathbb{C}}$ is the normalizer of $\mathrm{N}$, that gives the following equality

$$
\hat{d}^{-1}(z g) \hat{n}_{B}^{-1}(z g) \hat{n}(z) \hat{d}(z g)=\hat{n}(z, g), \quad \hat{n}(z, g) \in \mathrm{N} .
$$

Substituting $\hat{n}_{B}(z g) \hat{d}^{-1}(z g)$ for $\hat{d}^{-1}(z g) \hat{n}_{B}^{-1}(z g) \hat{n}(z)$ in (12) we obtain

$$
\hat{a}\left(z_{g}\right)=\hat{n}^{-1}\left(z_{g}\right) \hat{n}(z, g) \hat{d}^{-1}(z g) \hat{a}(z) \hat{k}(z) \hat{g} \hat{k}^{-1}\left(z_{g}\right) .
$$

To cancel the element $\hat{k}(z) \hat{g} \hat{k}^{-1}\left(z_{g}\right)=\hat{g}^{\prime} \in \mathrm{G}$ we take the following product

$$
\hat{a}^{2}\left(z_{g}\right)=\hat{a}\left(z_{g}\right) \hat{a}^{*}\left(z_{g}\right)=\hat{n} \hat{d}^{-1}\left(z_{g}\right) \hat{a}^{2}(z) \hat{d}^{*-1}\left(z_{g}\right) \hat{n}^{*},
$$

where $\hat{n}$ denotes $\hat{n}^{-1}\left(z_{g}\right) \hat{n}(z, g) \in \mathrm{N}$.

Now we construct a one-dimensional real representation of (13). Let $\chi^{\xi}(\hat{a})$ denote a representation of $\hat{a}$ with real weights $\xi=\left(\xi_{1}, \xi_{2}, \ldots, \xi_{l}\right)$. A one-dimensional real representation of $\hat{a} \in \mathrm{A}$ has the following form $\chi^{\xi}(\hat{a})=r_{1}^{\xi_{1}} r_{2}^{\xi_{2}} \cdots r_{l}^{\xi_{l}}$, and a one-dimensional real representation of $\hat{d} \in \mathrm{T}^{\mathbb{C}}$ has the form $\chi^{\xi}(\hat{d})=d_{1}^{\xi_{1}} d_{2}^{\xi_{2}} \cdots d_{l}^{\xi_{l}}$. Therefore, the representation of $\hat{a}^{2}\left(z_{g}\right)$ gets the form

$$
\chi^{2 \xi}\left(\hat{a}\left(z_{g}\right)\right)=\chi^{\xi}(\hat{d}(z g)) \overline{\chi^{\xi}(\hat{d}(z g))} \chi^{2 \xi}(\hat{a}(z)) .
$$

Whence it is seen that $\chi^{2 \xi}(\hat{a}(z))$ is transformed by a cocycle $\chi^{\xi}(\hat{d}(z g))$ defined on $\mathrm{G} \times \mathcal{O}$. It means that the function

$$
\ln \chi^{2 \xi}(\hat{a}(z))=\xi_{1} \ln r_{1}^{2}(z)+\xi_{2} \ln r_{2}^{2}(z)+\cdots+\xi_{l} \ln r_{l}^{2}(z)
$$

is G-covariant, and serves as a Kählerian potential on $\mathcal{O}$. Moreover, each function $\ln r_{i}^{2}(z), i=1 . . l$, is a Kählerian potential itself.

Remarkably, that each coadjoint orbit has a unique Kählerian potential of the form (14), where the weights $\xi=\left(\xi_{1}, \xi_{2}, \ldots, \xi_{l}\right)$ are determined by an initial point of the orbit. We have proven the following

Proposition 6. Suppose A is the real abelian subgroup of $\mathrm{G}^{\mathbb{C}}, \hat{a} \in \mathrm{A}$, and $\chi^{\xi}(\hat{a})$ is a one-dimensional representation of $\hat{a}$ with real weights $\xi=\left(\xi_{1}, \xi_{2}, \ldots, \xi_{l}\right)$. Then Kählerian potentials on coadjoint orbits of $\mathrm{G}$ have the form $\ln \chi^{2 \xi}(\hat{a})$, moreover each orbit has the Kählerian potential with a unique $\xi$.

Remark 1. In the case of integer weights $\xi=\left(\xi_{1}, \xi_{2}, \ldots, \xi_{l}\right)$, the line bundle over each coadjoint orbit of $\mathrm{G}$ is holomorphic. This idea is derived from the BorelWeyl theory based on [8].

Consider several examples.

Example 6. In the case of group $\mathrm{SU}(n)$, a representative of the real abelian subgroup A has the form of a diagonal matrix with $\operatorname{det} \hat{a}=1$, that is

$$
\hat{a}=\operatorname{diag}\left(1 / r_{1}, r_{1} / r_{2}, \ldots, r_{n-2} / r_{n-1}, r_{n-1}\right),
$$


and $\operatorname{dim} \mathrm{T}=n-1$. Let $\left(r_{1}, r_{2}, \ldots, r_{n-1}\right)$ be an image of $\hat{a}$ under an isomorphism from $\mathrm{T}^{\mathbb{C}}$ onto $\left(\mathbb{C}^{*}\right)^{n-1}$. Then $\chi^{\xi}(\hat{a})=r_{1}^{\xi_{1}} r_{2}^{\xi_{2}} \cdots r_{n-1}^{\xi_{n-1}}$, where $\xi_{i} \in \mathbb{R}$, $i=1$.. $(n-1)$, whence Kählerian potentials have the following form:

$$
\Phi=\xi_{1} \ln r_{1}^{2}+\xi_{2} \ln r_{2}^{2}+\cdots+\xi_{n-1} \ln r_{n-1}^{2} .
$$

For instance, Kählerian potentials on orbits of $\mathrm{SU}(3)$ are

$$
\begin{gathered}
\Phi=\xi \ln r_{1}^{2}+\eta \ln r_{2}^{2} \\
r_{1}^{2}=1+\left|z_{1}\right|^{2}+\left|z_{3}-z_{1} z_{2}\right|^{2}, \quad r_{2}^{2}=1+\left|z_{2}\right|^{2}+\left|z_{3}\right|^{2} .
\end{gathered}
$$

This expression completely accords with the straightforward solution of (8), which gives the following:

$$
\begin{gathered}
\Phi=\left\langle\hat{\mu}_{0}, \hat{\alpha}_{1}\right\rangle \Phi_{1}+\left\langle\hat{\mu}_{0}, \hat{\alpha}_{2}\right\rangle \Phi_{2} \\
\Phi_{1}=\ln \left(1+\left|z_{1}\right|^{2}+\left|z_{3}-z_{1} z_{2}\right|^{2}\right), \quad \Phi_{2}=\ln \left(1+\left|z_{2}\right|^{2}+\left|z_{3}\right|^{2}\right),
\end{gathered}
$$

here $\hat{\mu}_{0}$ is an initial point of an orbit, $\hat{\alpha}_{1}, \hat{\alpha}_{2}$ are the simple roots of $\mathfrak{s u}(3)$. In the case of a degenerate orbit, one has to assign $z_{1}=0$ or $z_{2}=0$.

Example 7. In the case of groups $\mathrm{SO}(n), n=2 m$ and $n=2 m+1$, a representative of the subgroup A has the form of a block-diagonal matrix, namely

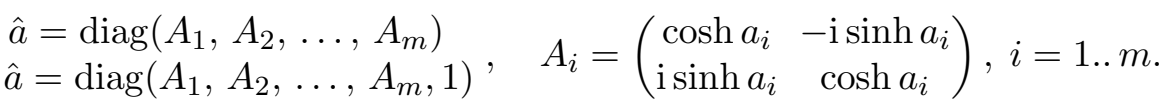

Here $\left\{a_{i}\right\}$ are canonical coordinates in the maximal torus $\mathrm{T}$, and $\operatorname{dim} \mathrm{T}=m$. Let $\left(e^{a_{1}}, e^{a_{2}}, \ldots, e^{a_{m}}\right)$ be an image of $\hat{a}$ under an isomorphism from $\mathrm{T}^{\mathbb{C}}$ onto $\left(\mathbb{C}^{*}\right)^{m}$. Then $\chi^{\xi}(\hat{a})=e^{\xi_{1} a_{1}} e^{\xi_{2} a_{2}} \cdots e^{\xi_{m} a_{m}}$, whence it follows

$$
\Phi=2 \xi_{1} a_{1}+2 \xi_{2} a_{2}+\cdots+2 \xi_{m} a_{m} .
$$

Kählerian potentials on coadjoint orbits of $\mathrm{SO}(4)$ computed by (8) have the form

$$
\begin{gathered}
\Phi=\left\langle\hat{\mu}_{0}, \hat{\alpha}_{1}\right\rangle \Phi_{1}+\left\langle\hat{\mu}_{0}, \hat{\alpha}_{2}\right\rangle \Phi_{2} \\
\Phi_{1}=\ln \left(1+\left|z_{1}\right|^{2}\right)-\ln \left(1+\left|z_{2}\right|^{2}\right), \quad \Phi_{2}=\ln \left(1+\left|z_{1}\right|^{2}\right)+\ln \left(1+\left|z_{2}\right|^{2}\right) .
\end{gathered}
$$

Here the bilinear form on $\mathfrak{s o}(4)$ is defined by $\langle A, B\rangle=\frac{1}{2} \operatorname{Tr} A B$.

Proposition 7. The Kählerian potential on each coadjoint orbit $\mathcal{O}_{\mu_{0}}$ of a compact classical Lie group $\mathrm{G}$ has the following form

$$
\Phi=\sum_{k}\left\langle\mu_{0}, \alpha_{k}\right\rangle \Phi_{k}, \quad \Phi_{k}=a_{\alpha_{k}},
$$

where $\alpha_{k}$ is a simple root of $\mathfrak{g}$, and $a_{\alpha_{k}}$ is the canonical coordinate corresponding to $H_{\alpha_{k}} \in \mathfrak{h}$, and $\langle\cdot, \cdot\rangle$ denotes a bilinear form on the dual space to $\mathfrak{g}$. 
Remark 2. If $\mu_{0}$ satisfies the integer condition

$$
2 \frac{\left\langle\mu_{0}, \alpha_{k}\right\rangle}{\left\langle\alpha_{k}, \alpha_{k}\right\rangle} \in \mathbb{Z},
$$

then the orbit through $\mu_{0}$ can be quantized. In other words, there exists an irreducible unitary representation of $\mathrm{G}$ in the space of holomorphic sections on the orbit. Each section serves as a quantum state.

\section{Cohomology Rings of Coadjoint Orbits}

In the last section we examine the cohomology rings of coadjoint orbits of compact semisimple Lie groups. A. Borel [9] proved that all forms of odd degrees on the orbit are precise. Therefore, we are interested in the forms of even degrees. In order to introduce a basis for the cohomology ring it is sufficient to find a basis for the cohomology group $H^{2}$.

In the case of a generic coadjoint orbit of a compact semisimple Lie group $\mathrm{G}$, the following formula is available

$$
b^{0}+b^{2}+\cdots+b^{2 n}=\operatorname{ord} \mathrm{W}(\mathrm{G}),
$$

where $b^{k}$ denotes the Betti number of a cohomology group $H^{k}$. In the case of a degenerate orbit, one has to modify the formula as

$$
b^{0}+b^{2}+\cdots+b^{2 m}=\frac{\text { ord } \mathrm{W}(\mathrm{G})}{\operatorname{ord} \mathrm{W}\left(\mathrm{G}_{\mu_{0}}\right)},
$$

where $\mathrm{G}_{\mu_{0}}$ is the stability subgroup at $\mu_{0}$.

Example 8. In the case of group $\mathrm{SU}(2)$, we have the only type of orbits: $\mathcal{O}^{\mathrm{SU}(2)}$ of dimension 2. The Weyl group $\mathrm{W}(\mathrm{SU}(2))$ also has dimension 2. Therefore, the cohomology ring consists of two cohomology groups, each of dimension 1 :

$$
H^{*}=H^{0} \oplus H^{2}, \quad 1+1=2 .
$$

In the case of group $\mathrm{SU}(3)$, we have two types of orbits: a generic one $\mathcal{O}^{\mathrm{SU}(3)}$ of dimension 6, and a degenerate one $\mathcal{O}_{d}^{\mathrm{SU}(3)}$ of dimension 4. In the case of a generic orbit, the Weyl group has dimension 6, and the cohomology ring is

$$
H^{*}=H^{0} \oplus H^{2} \oplus H^{4} \oplus H^{6}, \quad 1+2+2+1=6 .
$$

For a degenerate orbit we have $\frac{\text { ord } \mathrm{W}(\mathrm{G})}{\operatorname{ord} \mathrm{W}\left(\mathrm{G}_{\mu_{0}}\right)}=3$, and the cohomology ring is

$$
H^{*}=H^{0} \oplus H^{2} \oplus H^{4}, \quad 1+1+1=3 .
$$

Recall the well-known Leray-Hirsch theorem. 
Theorem (Leray-Hirsch). Suppose $\mathcal{E}$ is a fibre bundle over a base $\mathcal{M}$ with a fibre $\mathcal{F}$, and $\omega_{1}, \omega_{2}, \ldots \omega_{r}$ are cohomology classes on $\mathcal{E}$ that being restricted to each fibre give its cohomologies. Then

$$
H^{*}(\mathcal{E})=H^{*}(\mathcal{M}) \otimes H^{*}(\mathcal{F})
$$

Apply the theorem to an orbit $\mathcal{O}$ regarded as a fibre bundle over an orbit $\mathcal{O}_{1}$ with an orbit $\mathcal{O}_{2}$ as a fibre, that is $\mathcal{O}=\mathcal{E}\left(\mathcal{O}_{1}, \mathcal{O}_{2}, \pi\right)$. The cohomology ring of $\mathcal{O}$ is a tensor product of the cohomology rings of the base and the fiber:

$$
H^{*}(\mathcal{O})=H^{*}\left(\mathcal{O}_{1}\right) \otimes H^{*}\left(\mathcal{O}_{2}\right)
$$

Conversely, if one finds coherent cohomology classes on $\mathcal{O}_{1}$ and $\mathcal{O}_{2}$, then one can construct the cohomology ring of $\mathcal{O}$ by the latter formula. It means, the cohomology ring of a generic orbit can be deriven from the cohomology rings of a degenerate orbit and a generic orbit of a group of less dimension.

Example 9. We continue to deal with the group SU(3). It was shown that

$$
\mathcal{O}^{\mathrm{SU}(3)}=\mathcal{E}\left(\mathcal{O}_{d}^{\mathrm{SU}(3)}, \mathcal{O}^{\mathrm{SU}(2)}, \pi\right) .
$$

Then the cohomology ring of $\mathcal{O}^{\mathrm{SU}(3)}$ is the tensor product of the cohomology rings of the orbits $\mathcal{O}_{d}^{\mathrm{SU}(3)}$ and $\mathcal{O}^{\mathrm{SU}(2)}$ :

$$
\begin{aligned}
& H^{*}\left(\mathcal{O}^{\mathrm{SU}(3)}\right)=\left(H^{0} \oplus H^{2} \oplus H^{4}\right) \otimes\left(H^{0} \oplus H^{2}\right)= \\
& \quad=H^{0} \otimes H^{0} \oplus \underbrace{H^{0} \otimes H^{2} \oplus H^{2} \otimes H^{0}}_{H^{2}\left(\mathcal{O}^{\mathrm{SU}(3)}\right)} \oplus \underbrace{H^{2} \otimes H^{2} \oplus H^{4} \otimes H^{0}}_{H^{4}\left(\mathcal{O}^{\mathrm{SU}(3)}\right)} \oplus H^{4} \otimes H^{2} .
\end{aligned}
$$

Obviously, the cohomology groups $H^{2}$ and $H^{4}$ of $\mathcal{O}^{\mathrm{SU}(3)}$ both have dimension 2. Moreover, from the previous expression we can see the structure of a basis for $H^{2}$ :

$$
H^{2}\left(\mathcal{O}^{\mathrm{SU}(3)}\right)=H^{0}(1) \otimes H^{2}(2) \oplus H^{2}(1) \otimes H^{0}(2),
$$

where 1 denotes $\mathcal{O}_{d}^{\mathrm{SU}(3)} \simeq \mathbb{C P}^{2}$, and 2 denotes $\mathcal{O}^{\mathrm{SU}(2)} \simeq \mathbb{C P}^{1}$.

At the same time, a suitable basis for $H^{2}$ can be obtained from Kählerian potentials on coadjoint orbits of a group. As shown in the previous section, all two-forms on the orbits of a compact classical Lie group $\mathrm{G}$ have the form

$$
\omega=\sum_{k} \mathrm{i} c_{k} \sum_{\alpha, \beta} \frac{\partial^{2} \Phi_{k}}{\partial z_{\alpha} \partial \bar{z}_{\beta}} d z_{\alpha} \wedge d \bar{z}_{\beta}, \quad k=1, \ldots, \operatorname{dim} \mathrm{T},
$$

where $\Phi_{k}$ coincides with the canonical coordinate $a_{\alpha_{k}}$ corresponding to $H_{\alpha_{k}} \in \mathfrak{h}$. Obviously, $\operatorname{dim} H^{2}=\operatorname{dim} \mathrm{T}=l$. Consequently, one can find precisely $l$ twoforms that give a basis for $H^{2}$. 
The standard way to generate a basis for $H^{2}$ is the following. Let $H_{2}$ be the homology group adjoint to $H^{2}$. By $[\gamma]$ we denote a class of two-cycles, which can be represented as spheres. The sphere is an orbit of a subgroup $\mathrm{SU}_{\alpha}(2)$ :

$$
\mathrm{SU}_{\alpha}(2) \simeq \exp \left\{H_{\alpha},\left(X_{\alpha}-X_{-\alpha}\right), \mathrm{i}\left(X_{\alpha}+X_{-\alpha}\right)\right\}, \quad \alpha \in \Delta_{+} .
$$

Suppose we find $l$ independent two-cycles connected with the simple roots of $\mathfrak{g}$, we denote them by $\gamma_{i}$. The basis for $H^{2}$ consists of two-forms $\omega_{j}$ such that

$$
\int_{\gamma_{i}} \omega_{j}=\delta_{i j}
$$

where $\delta_{i j}$ is the Croneker symbol.

Example 10. We consider coadjoint orbits of $\mathrm{SU}(3)$ as an example. Let simple roots of $\mathfrak{s u}(3)$ be as follows: $\alpha_{1}=\operatorname{diag}(\mathrm{i},-\mathrm{i}, 0)$ and $\alpha_{2}=\operatorname{diag}(0, \mathrm{i},-\mathrm{i})$. Then independent two-cycles are generated by the following dressing matrices

$$
\hat{u}_{1}=\left(\begin{array}{ccc}
\frac{1}{\sqrt{1+\left|z_{1}\right|^{2}}} & \frac{-\bar{z}_{1}}{\sqrt{1+\left|z_{1}\right|^{2}}} & 0 \\
\frac{z_{1}}{\sqrt{1+\left|z_{1}\right|^{2}}} & \frac{1}{\sqrt{1+\left|z_{1}\right|^{2}}} & 0 \\
0 & 0 & 1
\end{array}\right), \quad \hat{u}_{2}=\left(\begin{array}{ccc}
1 & 0 & 0 \\
0 & \frac{1}{\sqrt{1+\left|z_{2}\right|^{2}}} & \frac{-\bar{z}_{2}}{\sqrt{1+\left|z_{2}\right|^{2}}} \\
0 & \frac{z_{2}}{\sqrt{1+\left|z_{2}\right|^{2}}} & \frac{1}{\sqrt{1+\left|z_{2}\right|^{2}}}
\end{array}\right),
$$

which are obtained from the dressing matrix $\hat{u}$ by assigning $z_{2}=z_{3}=0$ or $z_{1}=z_{3}=0$, respectively. The two-forms $\omega_{j}$ satisfying (15) are

$$
\begin{gathered}
\omega_{j}=\frac{1}{2 \pi} \sum_{\alpha, \beta} \frac{\partial^{2} \Phi_{j}}{\partial z_{\alpha} \partial \bar{z}_{\beta}} d z_{\alpha} \wedge d \bar{z}_{\beta}, \quad j=1,2 \\
\Phi_{1}=\ln \left(1+\left|z_{1}\right|^{2}+\left|z_{3}-z_{1} z_{2}\right|^{2}\right), \quad \Phi_{2}=\ln \left(1+\left|z_{2}\right|^{2}+\left|z_{3}\right|^{2}\right) .
\end{gathered}
$$

They form a basis for $H^{2}\left(\mathcal{O}^{\mathrm{SU}(3)}\right)$.

\section{Conclusion}

In this paper we develop a unified approach to solutions of the announced problems for a coadjoint orbit of a compact semisimple classical Lie group G. The problems are the following: an explicit parameterization of the orbit, obtaining a Kählerian structure, introducing basis forms for the cohomology group of the orbit. The key role belongs to the subgroup A in an Iwasawa decomposition, this is the real abelian subgroup of a complexification of the group G. The subgroup A determines a Kählerian potential on each orbit and a suitable basis for the cohomology group $H^{2}$ of the orbit.

Our investigation concerns classical (matrix) Lie groups. The same problems in the general case remain of current importance. 


\section{Acknowledgements}

We would like to thank professor A. Arvanitoyergos for fruitful debate and proper references, professor G. Vilasi for valuable discussion, professors I. Mladenov and M. Hadzhilazova for their hospitality.

This work was partly supported by grant DFFD.Ukr F16/457-2007

\section{References}

[1] Bott R. The Geometry and Representation Theory of Compact Lie Groups, In: Representation Theory of Lie Groups, London Mathematical Society Lecture Note Series, Cambridge Univ. Press, 34 (1979) 65-90.

[2] Skrypnyk T., Coadjoint Orbits of Compact Lie Groups and Generalized Stereographic Projection, Ukr. Math. J. 51 (1999) 1714-1718.

[3] Picken R. F. The Duistermaat-Heckman Integration Formula on Flag Manifolds, J. Math. Phys., 31 (1990) 616-638.

[4] Helgason S., Differential Geometry, Lie Groups and Symmetric Spaces, Academic Press, Toronto, 1978.

[5] Borel A. Kählerian Coset Spaces of Semisimple Lie Groups, Proceedings of the National Academy of Sciences of the United States of America 40 (1954), 1147-1151.

[6] Besse A. L. Einstein Manifolds, Springer-Verlag, 1987.

[7] Alekseevsky D. V., Perelomov A. M. Invariant Kähler-Einstein Metrics on Compact Hologenious Spaces, Functional Anal. Appl. 20 (1986) 171-182.

[8] Borel A., Weyl A. Representations Lineares et Espaces Homogenes Kählerians des Groupes de Lie Compacts, Seminaire Bourbaki, May 1954.

[9] Borel A. Sur la Cohomologie des Espaces Fibres Principaux et des Espaces Homogenes de Groups de Lie Compacts, Ann. Math. 57 (1953), 115-207. 